\title{
Simulation of Damage Evolution and Accumulation in Vanadium
}

\author{
E. Alonso \\ M.-J. Caturla \\ T. Díaz de la Rubia \\ J.M. Perlado
}

February 8, 1999

This is an informal report intended primarily for internal or limited external distribution. The opinions and conclusions stated are those of the author and may or may not be those of the Laboratory.

Work performed under the auspices of the U.S. Department of Energy by the Lawrence Livermore National Laboratory under Contract W-7405-ENG-48. 


\section{DISCLAIMER}

This document was prepared as an account of work sponsored by an agency of the United States Government. Neither the United States Government nor the University of California nor any of their employees, makes any warranty, express or implied, or assumes any legal liability or responsibility for the accuracy, completeness, or usefulness of any information, apparatus, product, or process disclosed, or represents that its use would not infringe privately owned rights. Reference herein to any specific commercial product, process, or service by trade name, trademark, manufacturer, or otherwise, does not necessarily constitute or imply its endorsement, recommendation, or favoring by the United States Government or the University of California. The views and opinions of authors expressed herein do not necessarily state or reflect those of the United States Government or the University of California, and shall not be used for advertising or product endorsement purposes.

This report has been reproduced directly from the best available copy.

Available to DOE and DOE contractors from the Office of Scientific and Technical Information P.O. Box 62, Oak Ridge, TN 37831

Prices available from (423) 576-8401

Available to the public from the National Technical Information Service

U.S. Department of Commerce 5285 Port Royal Rd., Springfield, VA 22161 


\title{
SIMULATION OF DAMAGE EVOLUTION AND ACCUMULATION IN VANADIUM
}

\author{
E. Alonso, M.-J. Caturla, T. Díaz de la Rubia \\ Lawrence Livermore National Laboratory, P.O. Box 808, \\ Livermore, CA 94550, USA \\ J.M. Perlado* \\ "Instituto de Fusión Nuclear, José Gutiérrez Abascal, 2 \\ E.T.S.I.I \\ 28006 Madrid, Spain
}

\begin{abstract}
Energetic atoms which have been knocked off their lattice sites by neutron or ion irradiation leave a trail of vacancies and interstitials in their wake. Most of these defects recombine with their opposites within their own collision cascade. Some fraction, however, escape to become freely migrating defects (FMD) in the bulk of the material. The interaction of FMD with the microstructure has long been linked to changes in the macroscopic properties of materials under irradiation. We calculate the fraction of FMD in pure vanadium for a wide range of temperatures and primary knock-on atom (PKA) energies. The collision cascade database is obtained from molecular dynamics (MD) simulations with an embedded atom method (EAM) potential. The actual FMD calculation is carried out by a kinetic Monte Carlo (kMC) code with a set of parameters extracted either from the experimental literature or from MD simulations. We take two different approaches to the problem and compare them. The first consists of an idealized simulation for single cascades. Annealing each cascade at different temperatures allows the mobile species to escape and account for FMD. The second analyzes bulk diffusion and damage
\end{abstract}


accumulation in a specimen irradiated at a low dose rate in the presence of impurities, in order to mimic experimental conditions. At the temperature studied, beginning of stage V, we observe that only vacancies are free to move whereas most interstitials are stopped by impurities. The fraction of FMD obtained is $11 \%$ for high purity vanadium, which is in good agreement with the figures reported in literature. We also analyze the role of impurities in damage accumulation.

\section{Introduction}

Vanadium-based alloys are of great technological importance for future fusion reactors [1]. Their low activation in a $14 \mathrm{MeV}$ neutron environment make them the ideal candidates for the structural material of the reactor first wall. Thus it is imperative that we fully understand the production and accumulation of radiation damage in these alloys. We present a study of damage production and accumulation in pure vanadium in a first step towards addressing this issue. .

Radiation damage production and accumulation in solids can be divided into two stages. In the production stage, the impinging particle gradually gives off its kinetic energy to the atoms of the lattice in the form of energetic recoils. These recoils deposit their energy in the lattice by generating secondary and higher order recoils that result in a displacement collision cascade. The outcome of this stage, of the time scale of ps, is a population of point or clustered defects known as the primary state of damage. In the second stage, which can extend over seconds, defects that survive recombination within their nascent cascade migrate over long distances, interacting with the microstructure. These freely migrating defects (FMD) are responsible for the changes in the macroscopic properties of metals under irradiation, such as void swelling, embrittlement, radiation enhanced diffusion, etc.

Computer simulation efforts have been conducted in an attempt to model the two stages of radiation damage. Molecular dynamics (MD) simulations studies using many- 
body potentials of the embedded atom method (EAM) type have proven to be very successful in the description of the first stage of damage production [2,3]. The modeling of the second stage has historically been undertaken by rate theory [4-6]. However, the application of kinetic Monte Carlo (kMC) simulations to diffusion processes [7,8] is starting to gain acceptance among the radiation damage community. The main strength of $\mathrm{kMC}$ stems from the fact that the highly inhomogeneous nature of the spatial and temporal damage distribution can be easily incorporated and treated. Moreover, features such as onedimensional migration of small interstitial clusters can also be treated within the same model.

One quantity of fundamental interest for understanding radiation effects in solids is the fraction of FMD. This fraction has been obtained by different experimental methods in the literature. The main approach has been to analyze changes in macroscopic properties believed to depend on the FMD population and to interpret these changes in terms of rate equations. Measurements of radiation-enhanced diffusion [9-13], radiation-induced segregation [14-16], dislocation pinning [17], swelling rates [18], ordering rates [19] and electrical resistivity [20] have been used during the past twenty years to estimate this fraction.

We present a calculation of the fraction of FMD in vanadium obtained by coupling MD with kMC simulations. We compare our results with the experimental data reported in the literature. We carry out two kinds of calculation. In the first, a single cascade is annealed at various temperatures. This represents the dilute upper limit of the FMD fraction corresponding to very low dose irradiation conditions. The second is a simulation of damage accumulation at a fixed dose rate and temperature with varying impurity concentration. For the sake of argument the impurities will be considered to be traps of infinite strength for interstitials and transparent to vacancies. The role of impurities will be analyzed in terms of FMD population and damage accumulation behavior. 


\section{Cascade simulations and defect production}

We simulate the primary state of damage by MD simulations. In order to obtain reliable statistics a significant number of cascades has to be produced for each energy. The details of these simulations are shown in table 1. All MD simulations were performed using the MDCASK code either in its parallel or serial version. All the collision cascades were simulated at room temperature $(300 \mathrm{~K})$. Since little influence of the irradiation temperature on defect production has been observed [2,21,22], the defect configurations so generated were used along the entire temperature range investigated by $\mathrm{kMC}$. The temperature was controlled by connecting the MD box to a thermal bath applying Langevin dynamics [23] to the two outer layers of atoms. The atomic interactions were described by the Johnson and Oh EAM potential [24]. The short range interaction part was smoothly splined to the universal potential of Biersack and Ziegler [25]. Previous studies with this potential have accurately reproduced the melting point and the displacement threshold energies [26].

Due to the open character of the bcc structure, channeling occurred in most of the high energy cascades. Simulations in which channeling caused a cascade to cross the boundaries of the simulation box were eliminated. Only three of the $20 \mathrm{keV}$ cascades were completed out of more than ten started. The simulations were continued until the number of defects reached a stable population. The high melting point of vanadium confines the thermal spike associated with the cascade to a small region that cools down quickly. Figure 1 shows the configuration of the damage produced by a $10 \mathrm{keV}$ recoil after $15 \mathrm{ps}$. The light points correspond to self-interstitial atoms and the dark ones to vacancies. Although vacancies occupy the central part and SIAs the periphery, the trend is not so obvious as in fcc metals. The defects are spread over the simulation box mostly as point defects. Only small interstitial and vacancy clusters are formed during this stage.

An important result of these simulations is the low interstitial and vacancy clustering observed. We consider a defect cluster to be any group of defects in which each of its 
members has at least one of the other elements of the cluster in a first nearest neighbor position. Figure 2 shows the interstitial cluster distribution as a function of recoil energy and cluster size. The largest cluster obtained was of 7 units in a $2 \mathrm{keV}$ cascade. Except for this cluster, no clusters greater than 3 were observed in cascades below $10 \mathrm{keV}$. This low clustering is in good agreement with previous MD simulations in bcc metals [21,22,26-28].

Another result of these simulations is the effectiveness of the thermal spike in repairing damage, in contrast to the predictions of a purely ballistic model [29]. Figure 3 depicts the efficiency of the ballistic NRT model to describe damage production. The collisional models break down at high energies because of the local melting of the material $[30,31]$ and the damage production saturates at high energies due to subcascade formation. The efficiency value obtained at high energy recoils is $26 \%$, in good agreement with previous work in bcc $[21,22,27,28]$.

\section{Kinetic Monte Carlo simulations}

In order to extend the time scale of the simulation we couple the MD output to a kMC code. The kMC simulations were performed with the BigMac code written by Mark Johnson at LLNL. The details of its operation are described elsewhere [32]. The input needed for the code is the primary state of damage obtained from MD, the diffusivities of the single and clustered defects, and the binding energies of the defect clusters. The input parameters of the model are shown in table 2. All vacancy clusters are assumed to be immobile. The binding energy of vacancy clusters was computed by MD. The formation energy of different cluster configurations was calculated up to size 6 minimizing the energy by a quasi-newton method [33]. A fit of this curve was made to determine the binding energy as a function of cluster size for larger clusters. The main assumption was that the most stable configuration of large vacancy clusters is purely spherical. Although in pure metals a void will collapse to form a faulted dislocation loop, the presence of helium 
stabilizes spherical voids. Therefore the assumption should hold for vanadium, a material with high impurity content and in a helium producing environment. Thus another point of the curve could be computed for a better fit at large sizes. One perfectly spherical void containing more than 100 atoms was relaxed and its formation energy calculated. As expected under this assumption, the best fit of the formation energy as a function of cluster size is close to a power of $(2 / 3)$.

The interstitial cluster diffusivities and binding energies are those calculated by Naoki Soneda for iron with the Johnson\&Oh potential [26]. Iron and vanadium have a number of important similarities: the same type of lattice and proximity in the periodic table. Moreover, within the context of these calculations both metals are described by the same type of interatomic potential. This factor in particular led us to presume that a detailed study of cluster binding energies and cluster diffusivities in vanadium would yield similar numbers to those of iron. Single-interstitials and interstitial clusters of sizes up to 3 were allowed to migrate three-dimensionally. Interstitial clusters of sizes between 4 and 19 were constrained to one-dimensional migration (glissile) and larger clusters were considered to be sessile.

\section{Freely migrating defects from single cascades}

FMD are those defects that escape recombination within their collision cascade. In such a case, the outgoing flux of defects through a spherical surface centered at the cascade and of sufficient radius will account for those defects that are highly unlikely to undergo recombination. Obviously this definition is sensitive to the radius of the sphere, the simulation time and the temperature. In a real irradiation experiment cascade overlapping occurs, so it is reasonable to set a simulation time slightly lower than the time for cascade overlapping for a given dose rate. Since the simulation time is fixed by the dose rate and the temperature is another physical magnitude, the only parameter of our model that can affect 
the FMD so calculated is the sphere radius. Due to the details of the $\mathrm{kMC}$ code, the simulation box is cubic. We compute the fraction of FMD as a function of temperature and recoil energy and analyze the sensitivity of the model to the $\mathrm{kMC}$ box size.

The simulation details are as follows. We anneal the primary state of damage obtained in each of the cascades of our database in a $\mathrm{kMC}$ box of $100 \mathrm{~nm}$ edge. Each of the defects that leave the box are flagged as FMD and removed from the simulation. The simulation time is set to $1000 \mathrm{~s}$ which is equivalent to a dose rate of $10^{-9} \mathrm{~s}^{-1} \mathrm{~cm}^{-2}$ in a $100 \mathrm{~nm}$ box. In order to improve the statistical validity of the simulation, the calculation was repeated for 100 different random number sequences for each cascade. The same procedure was followed over the temperature range from $150 \mathrm{~K}$ to $750 \mathrm{~K}$. The fraction of freely migrating interstitials is displayed in figure 4 as a function of annealing temperature and PKA energy and the fraction of freely migrating vacancies in figure 5. The values shown are averaged over all the cascades available for each recoil energy.

As was shown in the previous section, the largest interstitial cluster found is of size 7, which is still mobile under our assumption. The high mobility of these clusters causes them all to leave the box over the entire range of temperatures investigated, as seen in figure 4. Another interesting feature of this simulation is the correlation between recoil energy and escape ratio. The higher the PKA energy, the more defects produced, therefore the higher the probability for those defects to recombine and the lower the escape ratio. This observation is in contrast to similar work on other metals in which the opposite or no dependence were seen [34]. The type of lattice may play an important role in this difference. In the referenced study, the material analyzed was gold, an fcc metal. In fcc metals big dislocation loops are formed after the collision cascade and the vacancy rich core is clearly separated from the interstitials in the periphery.

The picture is considerably different for vacancies as shown in figure 5. The low diffusivity of the single vacancy means its motion is not noticeable until temperatures of about $270 \mathrm{~K}$, which is 100 degrees above the experimental temperature of stage III in this 
metal [35]. Stage III temperature is determined by the migration energy of the single vacancy. Unfortunately, the high impurity content of vanadium reflects in wide disagreements over the experimental temperatures of the annealing stages. Moreover, the annealing stages are more tied to impurity migration phenomena than to vacancy migration [36]. These factors led us to use the migration energy calculated by Bacon et al. [37] in the input parameters. Its value of $0.7 \mathrm{eV}$ seems like a good compromise given that experiments report values from $0.5 \mathrm{eV}$ to $1.2 \mathrm{eV}$ [35]. Therefore, the 100 degree shift of stage III is a direct consequence of our vacancy migration energy choice. For low energy recoils that is the only feature of the curve due to the absence of immobile vacancy clusters. At high PKA energies only the single vacancies have escaped after stage III has been reached, but small vacancy clusters still remain in the box. Only when the temperature is high enough for those clusters to break up and emit single vacancies can all the defects escape the box. This happens in stage $\mathrm{V}$, which occurs at the second step located at $550 \mathrm{~K}$ in the curves corresponding to the $5 \mathrm{keV}, 10 \mathrm{keV}$ and $20 \mathrm{keV}$ recoils. Once again, this temperature is 100 degrees above the experimental one [36].

The main conclusion of this study is that no production bias of vacancies occurs at any of the temperatures simulated. The fraction of FMD obtained for $20 \mathrm{keV}$ recoils is $17 \%$ after normalizing by the NRT model. This number is in the same line of previous computer simulations of metals $[28,34,38]$.

The sensitivity of the FMD to the kMC box size was studied. The same simulation was performed for one of the $20 \mathrm{keV}$ cascades, varying the edge of the simulation box from $50 \mathrm{~nm}$ to $200 \mathrm{~nm}$. No appreciable difference in the number of FMD was found. The only difference observed was at the stage III transition temperature. In that temperature regime, the motion of single-vacancies is extremely low and the box size may slightly shift the transition temperature. Some slowly moving vacancies escaped from the small boxes while they did not have enough time in the big ones. This effect is completely negligible. 


\section{Damage accumulation}

The simulation of the previous section is purely ideal and no experiment would ever measure that FMD concentration. The interaction of newly produced point defects with the damage from previous cascades will dramatically lower that figure. In an attempt to calculate the fraction of FMD in a more realistic fashion we perform the following damage accumulation simulation:

- Dose rate of $10^{-4} \mathrm{dpa} / \mathrm{s}$ in a $\mathrm{kMC}$ box of $200 \mathrm{~nm}$ edge.

- Periodic boundary conditions along the three coordinate axes.

- Flat recoil spectrum of $20 \mathrm{keV}$. The fraction of FMD represented by $\eta$ is calculated as follows:

$$
\eta=\frac{\mathrm{N}_{\mathrm{FMD}}}{\mathrm{NRT}}
$$

The fraction of FMD so calculated is independent of recoil energy once the NRT model efficiency is constant. Figure 3 shows that the efficiency is already constant at 20 $\mathrm{keV}$. Therefore, the results are directly comparable to experiments of neutron or heavy ions irradiation.

- Temperature of $650 \mathrm{~K}$, well above the transition temperature for stage $\mathrm{V}$ where vacancies clusters become unstable.

- Two spherical sinks with a capture radius of $1.5 \mathrm{~nm}$, both for interstitial and vacancies. These long range sinks play a role similar to that of dislocations. Assuming an effective length for the dislocation of the order of the $\mathrm{kMC}$ box the dislocation density is $5 \cdot 10^{9} \mathrm{dis} / \mathrm{cm}^{2}$.

- Presence of interstitial traps. 
The traps can be likened to generic impurities. They are immobile, which reproduces a stable population of impurities once steady-state is reached. They are transparent to vacancies but form a tightly bound complex with single-interstitials. In other words, they are sinks of infinite strength for single-interstitials. Once the impurity-interstitial complex is formed, the arrival of new interstitials can make it grow but it will always remain infinitely bound. The only way such complexes can shrink is by the arrival of diffusing vacancies.

In a simulation of this sort, the number of FMD equals the number of mobile species immediately before the introduction of a new collision cascade and subsequent defect overlap. The population of mobile species will drop noticeably after several cascades. More defects will be available for recombination and a steady count of FMD will be reached. The fraction of FMD will be this steady-state value over the number of defects produced by the recoil energy analyzed according to the NRT model.

The absence of detrapping will have a negligible effect on the fraction of FMD. On the other hand, its influence on the damage accumulation will not allow us to establish quantitative comparisons with experiments. The no-detrapping feature speeds up the simulation driving the system towards the saturation of the damage at low doses. Despite this mismatch between damage and dose, qualitative conclusions can be drawn since the overall trend is preserved.

We repeat the calculation for two different impurity concentrations: 5 ppm and 100 ppm. In this way we can single out the role of impurities both on the fraction of FMD and on the damage accumulation. The simulation with $5 \mathrm{ppm}$ is clearly more representative of a high purity sample used by experimentalists.

Figures 6 and 7 show how the damage accumulation occurs in both simulations. Damage saturation is quickly reached at about $10^{-3} \mathrm{dpa}$ in the high purity case. The cluster density in the 100 ppm case keeps increasing linearly at the same dose. These curves are not quantitatively comparable to experiments, not only for the lack of detrapping previously 
mentioned, but also due to the small size of the clusters in the simulation. As will be explained later, none of the clusters in the simulation should be visible in the microscope.

The different behavior of the two simulations is easy to explain in terms of occupied trapping sites and free defects available. Only when the vast majority of the interstitial traps have captured at least one interstitial, can cluster growth be expected. Once this trap saturation is reached the cluster density growth drops departing from linear behavior and the growth of the already existing clusters takes place. Evidently, in the high purity case, the lower concentration of trap sites and consequent faster saturation of them leads to a saturation of the damage faster than in the low purity case. The number of trap sites not only affects the damage accumulation behavior, but also the number of freely migrating defects. Most of the cascade produced interstitials are promptly captured by impurities leaving a population of freely migrating interstitials close to zero. In any case, the dependence of this rather small number of freely migrating interstitials on the number of traps is beyond all doubt. The trap concentration plays its role on the number of freely migrating vacancies also. Although trapping sites are transparent to vacancies, saturated traps are an effective sink for them. This dependence is shown in figures 8 and 9. As seen in figure 8 , the average of the population of single vacancies in the instant before the next recoil entrance is 20 in the case of $5 \mathrm{ppm}$ of impurities. This average is obtained only over the points of the curve with a steady population of free vacancies. Normalizing by the NRT collisional model we obtain a fraction of freely migrating vacancies of $11 \%$. In the low purity case of figure 9 , the number of freely migrating vacancies is $4,2.2 \%$ of the defects predicted by the NRT model. These numbers are in line with previous experimental and computer simulation results and display the strong dependence of the fraction of FMD and the concentration of impurities[13,28,34,38]. Due to the greater effectiveness of the traps at capturing interstitials, the fraction of freely migrating interstitials is much lower than the fraction of freely migrating vacancies for the two impurity concentrations. In the case of 5 ppm, the fraction of freely migrating interstitials is $1 \%$ and for $100 \mathrm{ppm}$ an almost 
negligible $0.07 \%$. Very likely, the presence of vacancy traps would sway these figures towards more balanced numbers both for freely migrating vacancies and interstitials. Nonetheless, the imbalance of the two populations of FMD shows the influence of the type of impurity on the fraction of FMD.

Figures 10 and 11 show the cluster size distribution function at two different doses

for the $5 \mathrm{ppm}$ case. At $10^{-3} \mathrm{dpa}$, a clear change in the shape of the curve is observed. A shift toward higher cluster sizes is shown in figure 11. Since TEM resolution is of the order of nanometers, only clusters over size 40 are visible. Since the largest clusters are of size 27, none of the clusters in our simulation would be visible in the microscope, as pointed out in the beginning of this section. In the case of the simulation with $100 \mathrm{ppm}$, since at $10^{-3} \mathrm{dpa}$ the damage accumulation is still in the linear regime, no cluster growth has occurred. The distribution function is not displayed in this case, because no significant changes in the form of the curve can be seen in the dose range analyzed.

\section{Conclusions}

We present an extensive study of damage accumulation coupling MD to simulate damage production and $\mathrm{kMC}$ to reproduce damage evolution. The collision cascades showed the expected low clustering behavior of bcc metals. However, the defect production obtained is lower than in the $5 \mathrm{keV}$ cascades at $10 \mathrm{~K}$ reported by Morishita et al. [26]. The production of self-interstitial atoms (SIA) drops dramatically from 18 at $10 \mathrm{~K}$ to 12 at $300 \mathrm{~K}$, which implies a 33\% decrease. Still, this is a small difference for such a large range in temperatures investigated and is therefore in good agreement with the no-temperaturedependence of damage production.

We calculate the fraction of FMD by two different approaches and compare both results. The first one is comparable to an infinitely small dose rate in a perfectly pure material. The second one consists of the simulation of low dose rate irradiation conditions 
in two different materials: one of high and another of low purity. The main difference detected is the imbalance in the number of freely migrating interstitials and freely migrating vacancies in the two cases with impurities. This disproportion is the result of the biased nature of the trapping sites. Therefore, different types of imbalances can be expected for different kinds of impurities. The absence of impurities evens out this imbalance as seen in the single-cascade simulation. There is a strong correlation between the impurity content and the fraction of FMD. For vacancies, this fraction is $17 \%$ in the pure case, drops down to $11 \%$ for only $5 \mathrm{ppm}$ of interstitial-trapping impurities and decreases to $2.2 \%$ for $100 \mathrm{ppm}$. We believe the damage accumulation simulation is the most valid method to compute the fraction of FMD.

We also analyze the role of impurities in the damage accumulation process. In spite of the impossibility of establishing direct comparison with experiments, qualitative conclusions can be drawn. The existence of impurities clearly increases the dose required to achieve the saturation of the damage. This saturation is reached in a state in which defect production and recombination are perfectly balanced. Until most of the trapping sites are taken, the trapping contribution is too high to allow equilibrium. Thus, the higher the content of impurities, the later the material will reach the steady state of damage.

In order to get a deeper insight into these phenomena and to reproduce experimental conditions in a more realistic manner, work is underway to include the effect of detrapping, real impurities and alloying elements.

\section{References}

[1] H.M. Chung, B.A. Loomis, D.L. Smith, J. Nucl. Mater. 239 (1996) 139.

[2] D.J. Bacon, T. Díaz de la Rubia, J. Nucl. Mater. 216 (1994) 275.

[3] R.S. Averback, J. Nucl. Mater. 216 (1994) 49. 
[4] R. Bullough, Proc. Conf. on Dislocations and Properties of Real Materials, Royal Society, London (The Institute of Metals, London, 1985) p. 382.

[5] H. Trinkaus, B.N. Singh, A.J.E. Foreman, J. Nucl. Mater. 249 (1997) 91.

[6] C.H. Woo, B.N. Singh, A.A. Semenov, J. Nucl. Mater. 239 (1996) 7.

[7] D.G. Doran, Rad. Effects 2 (1970) 249.

[8] H. L. Heinisch, J. Nucl. Mater. 117 (1983) 46.

[9] A. Müller, V. Naundorf, M.-P. Macht, J. Appl. Phys. 64 (1988) 3445

[10] V. Naundorf, M.-P. Macht, H. Wollenberger, J. Nucl. Mater. 186 (1992) 227.

[11] A. Iwase, L.E. Rehn, P.M. Baldo, L. Funk, Appl. Phys. Lett. 67 (1995) 229.

[12] P. Fielitz, M.-P. Macht, V. Naundorf, H. Wollenberger, Appl. Phys. Lett. 69 (1996) 331.

[13] P. Fielitz, M.-P. Macht, V. Naundorf, H. Wollenberger, Z. Metallkd. 87 (1996) 439.

[14] L.E. Rehn. P.R. Okamoto, R.S. Averback, Phys. Rev. B 30 (1984) 3073.

[15] T. Hashimoto, L.E. Rehn, P.R. Okamoto, Phys. Rev. B 38 (1988) 12865.

[16] R.A. Erck, L.E. Rehn, J. Nucl. Mater. 168 (1989) 208.

[17] J.A. Goldstone, D.M. Parkin, H.M. Simpson, J. Appl. Phys. 53 (1982) 4189.

[18] S.J. Zinkle, B.N. Singh, J. Nucl. Mater. 199 (1993) 173.

[19] M.A. Kirk, T.H. Blewitt. Metall. Trans. 9 (1978) 1729.

[20] S.J. Zinkle, J. Nucl. Mater. 155-157 (1988) 1201-1204.

[21] D.J. Bacon, A.F. Calder, F. Gao, V.G. Kapinos, S.J. Wooding, Nucl. Inst. and Meth. in Phys. Res. B, 102 (1995) 37.

[22] R.E. Stoller, J. Nucl. Mater. 233-237 (1996) 999.

[23] R. Biswas, D.R. Hamann, Phys. Rev. B 34 (1986) 895

[24] R.A. Johnson, D.J. Oh. J. Mater. Res. 4 (1989) 1195.

[25] W.D. Wilson, L.G. Haggmark, J.P. Biersack, Phys. Rev. B 15 (1977) 2458

[26] K. Morishita, T. Díaz de la Rubia, Mat. Res. Soc. Symp. Proc. 396 (1996) 39. 
[27] W.J. Phythian, R.E. Stoller, A.J.E. Foreman, A.F. Calder, D.J. Bacon, J. Nucl. Mater. 223 (1995) 245.

[28] N. Soneda, T Díaz de la Rubia, Phil Mag A 78 N-5 (1998) 995.

[29] M.J. Norgett, M.T. Robinson, I.M. Torrens, Nucl. Eng. Design 33 (1975) 50.

[30] F. Seitz, J.S. Koehler, in: Solid State Phys, eds. F. Seitz and D. Turnbull (Academic, New York, 1956), Vol 2.

[31] T. Diaz de la Rubia, R.S. Averback, R. Benedek, W.E. King, Phys. Rev. Lett. 59 (1987) 1930.

[32] M.D. Johnson, M.-J Caturla, T. Díaz de la Rubia, J. Appl. Phys. 84 (1998) 1963.

[33] J.B. Gibson, A.N. Goland, M. Milgram, G.H. Vineyard, Phys. Rev. 120 (1960) 1229.

[34] T.Díaz de la Rubia, N. Soneda, M.J. Caturla, E. Alonso, J. Nucl. Mater. 251 (1997) 13.

[35] H. Schultz, Landolt-Börnstein New Series III/25 (1991) 115.

[36] T. Leguey, R. Pareja, E.R. Hodgson, J. Nucl. Mater. 231 (1996) 191.

[37] D.J. Bacon, J.M. Harder, J. Nucl. Mater. 155-157 (1988) 1254.

[38] H.L. Heinisch, Rad. Eff. Def. Sol. 113 (1990) 53.

\section{Figures}

Figure 1. State of damage generated by a $10 \mathrm{keV}$ PKA at $300 \mathrm{~K}$. The light points represent self-interstitial atoms and the dark ones vacancies. The defect population is stable $15 \mathrm{ps}$ after the PKA entrance. No clusters larger than size 4 are formed. 
Figure 2. Average number of interstitial clusters as a function of size and PKA energy. The low clustering tendency is the same for vacancy clusters (Not shown in figure).

Figure 3. Efficiency of the NRT model as a function of PKA energy. The small drop from $5 \mathrm{keV}(34 \%)$ to $20 \mathrm{keV}(26 \%)$ indicates that the NRT efficiency is close to saturation.

Figure 4. Escape ratio of interstitials as a function of recoil energy and annealing temperature. Mobile interstitial clusters are also included in the escape ratio. Observe that the escape ratio decreases when the recoil energy increases.

Figure 5. Escape ratio of vacancies as a function of recoil energy and annealing temperature. The second step corresponding to stage V starts to be visible at PKA energies of more than $1 \mathrm{keV}$.

Figure 6. Interstitial and vacancy cluster density as a function of dose for vanadium with 5 ppm impurity content. All the interstitial clusters represented are trapped at the impurities. The number of free interstitial clusters is negligible.

Figure 7. Interstitial and vacancy cluster density as a function of dose for vanadium with $100 \mathrm{ppm}$ impurity content. The damage has not saturated at $10^{-3} \mathrm{dpa}$ because not all the trapping sites have been taken.

Figure 8. Number of freely migrating vacancies as a function of dose for vanadium with 5 ppm impurity content. The average number is 20 .

Figure 9. Number of freely migrating vacancies as a function of the dose for vanadium with 100 ppm impurity content. The average number is 4 vacancies. 
Figure 10. Cluster size distribution at $10^{-4} \mathrm{dpa}$ in vanadium with $5 \mathrm{ppm}$ impurity content.

Figure 11. Cluster size distribution at $10^{-3}$ dpa in vanadium with 5 ppm impurity content. Cluster growth has taken place after the trapping sites have been saturated. 
Table 1: Computational box size and number of simulations for each recoil energy.

\begin{tabular}{llll}
\hline Energy $(\mathrm{eV})$ & Box size (lat units) & no. of atoms & no. of cascades \\
\hline 20000 & $80 \times 80 \times 80$ & 1024000 & 3 \\
\hline 10000 & $50 \times 50 \times 50$ & 250000 & 6 \\
\hline 5000 & $45 \times 45 \times 45$ & 182250 & 4 \\
\hline 2000 & $45 \times 45 \times 45$ & 182250 & 7 \\
\hline 1000 & $30 \times 30 \times 30$ & 54000 & 9 \\
\hline 500 & $25 \times 25 \times 25$ & 31250 & 15 \\
\hline 200 & $25 \times 25 \times 25$ & 31250 & 15 \\
\hline 100 & $25 \times 25 \times 25$ & 31250 & 15 \\
\hline
\end{tabular}


Table 2: Input diffusivities for kMC and reference.

\begin{tabular}{llll}
\hline & $\mathrm{D}\left(\mathrm{cm}^{2} / \mathrm{s}\right)$ & $\mathrm{E}_{\mathrm{m}}(\mathrm{eV})$ & Reference \\
\hline $\mathrm{I}$ & $1.0 \mathrm{e}-03$ & 0.03 & {$[28]$} \\
\hline $\mathrm{I} 2$ & $7.5 \mathrm{e}-05$ & 0.067 & {$[28]$} \\
\hline $\mathrm{I} 3-\mathrm{I} 19$ & $1.4 \mathrm{e}-04$ & 0.17 & {$[28]$.} \\
\hline $\mathrm{V}$ & $1.0 \mathrm{e}-03$ & 0.7 & {$[37]$} \\
\hline
\end{tabular}


Figure 1

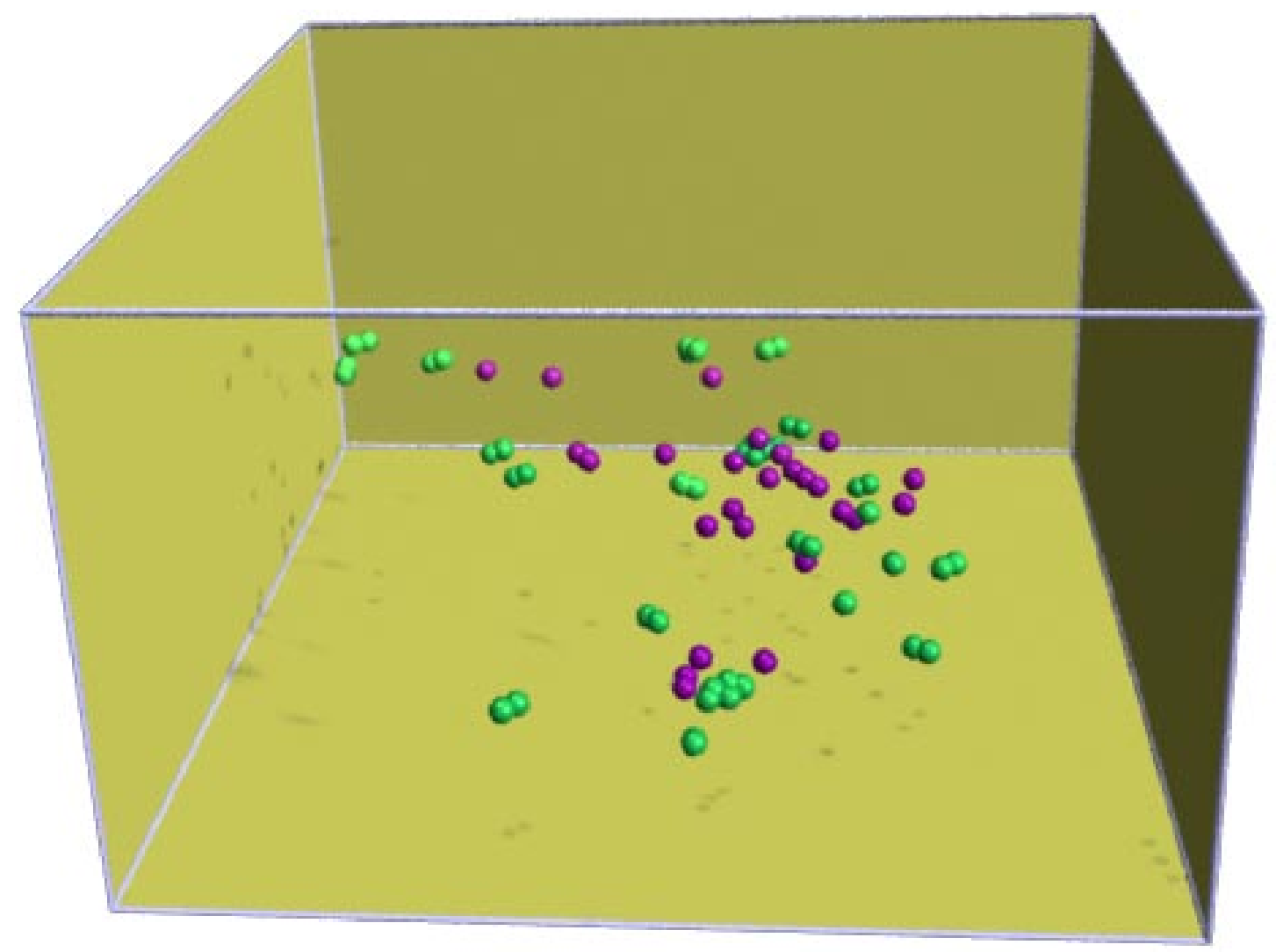


Figure 2.

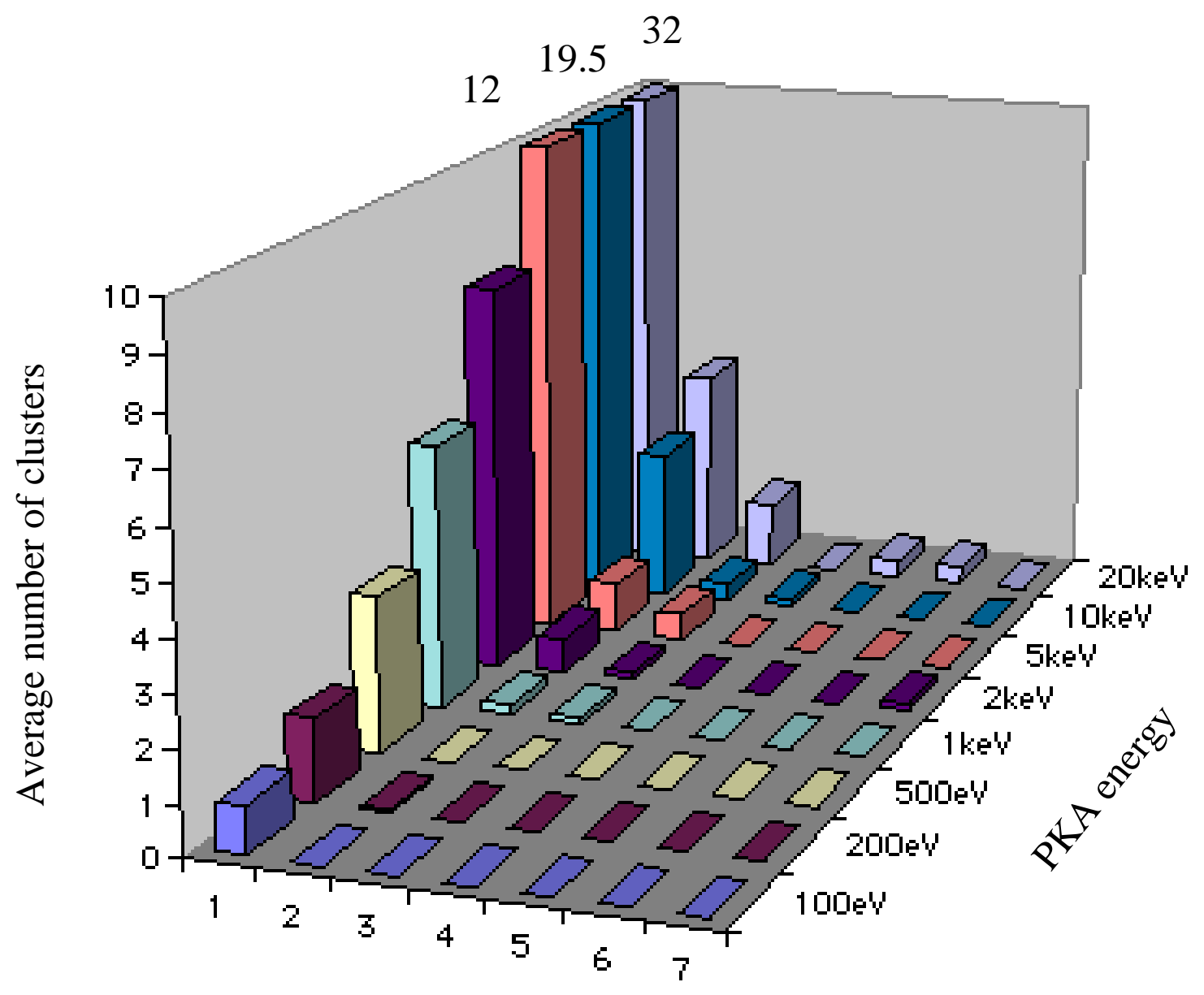

Cluster size 
Figure 3.

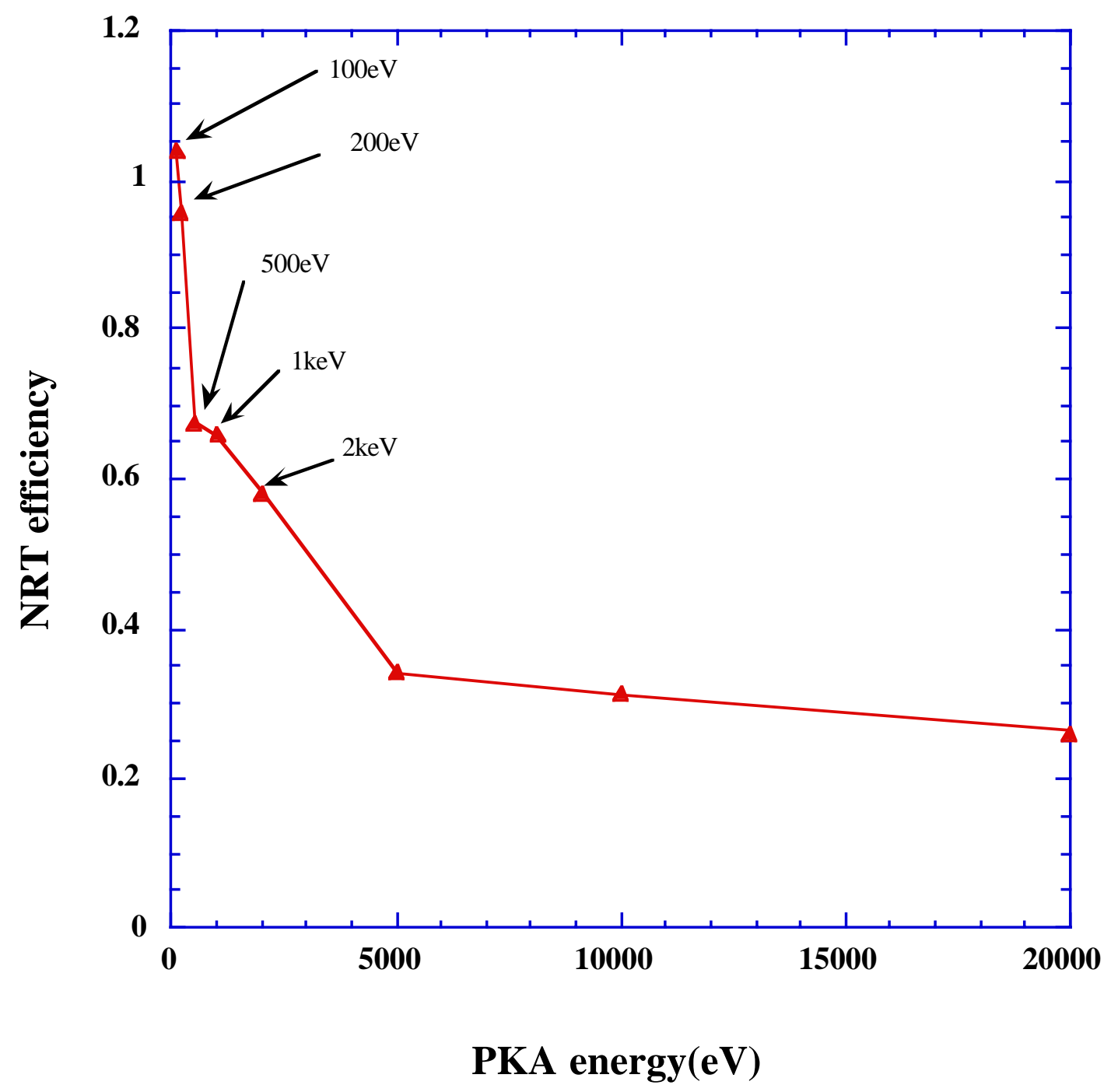


Figure 4.

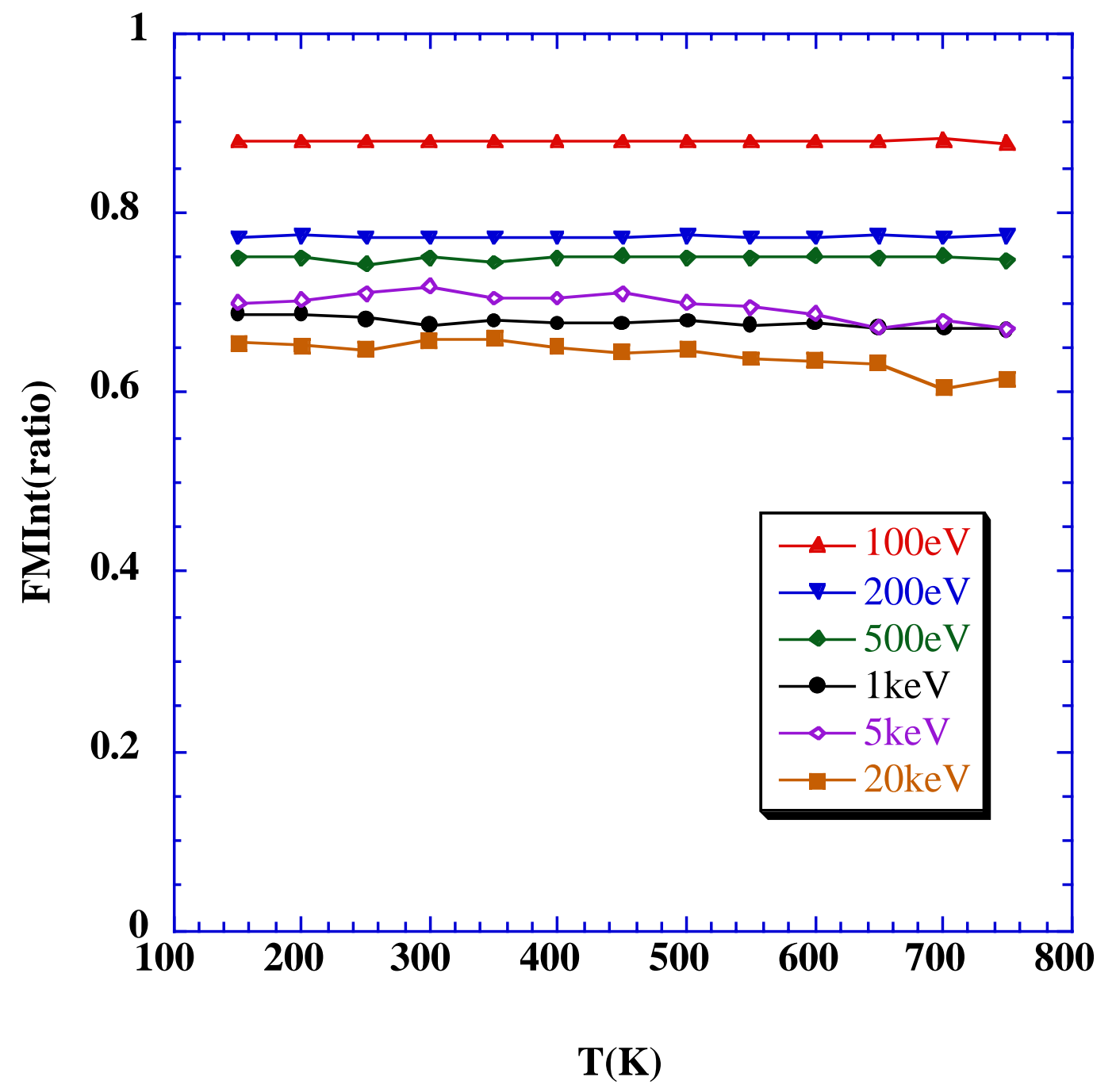

Figure 5. 


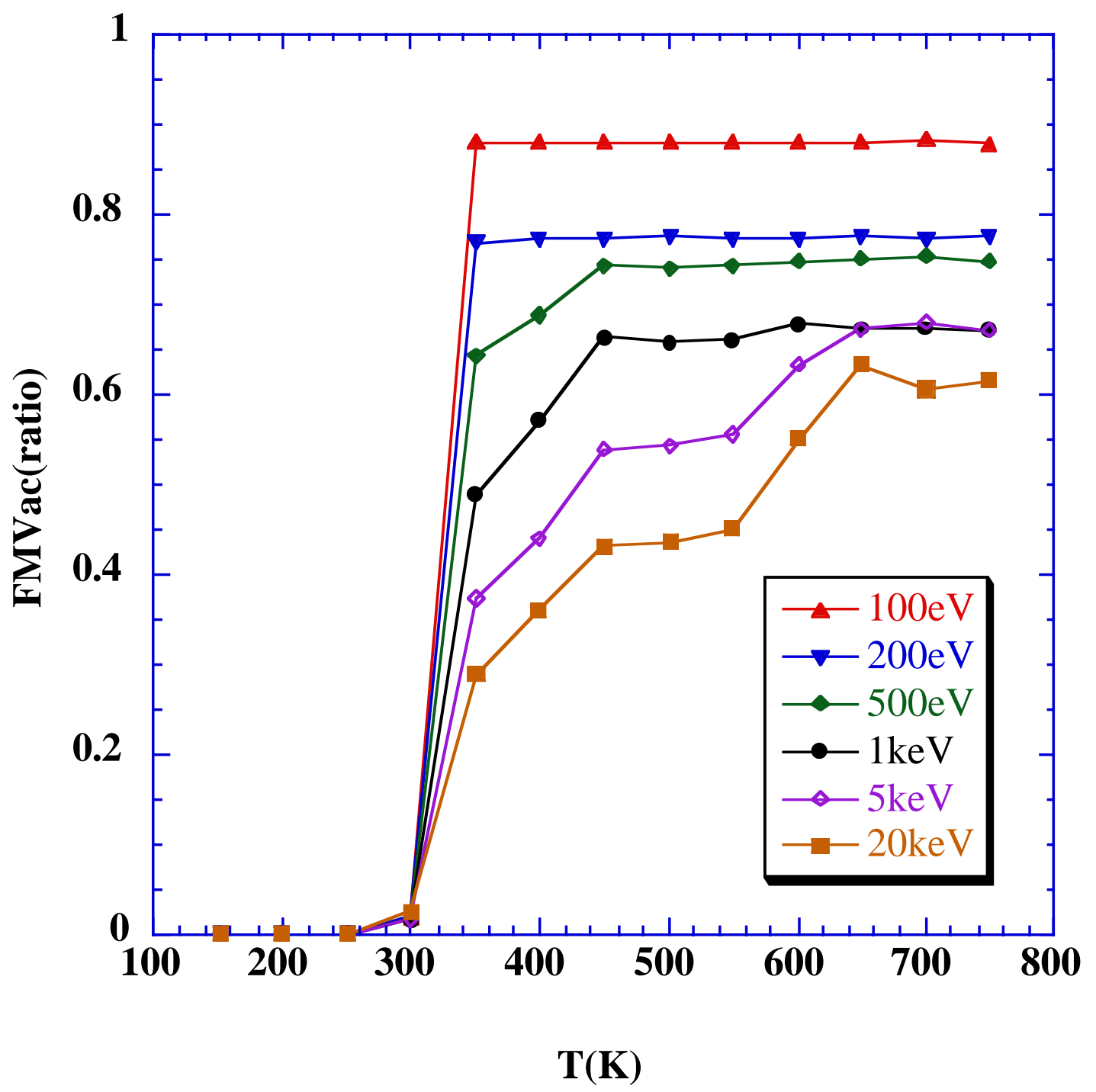

Figure 6. 


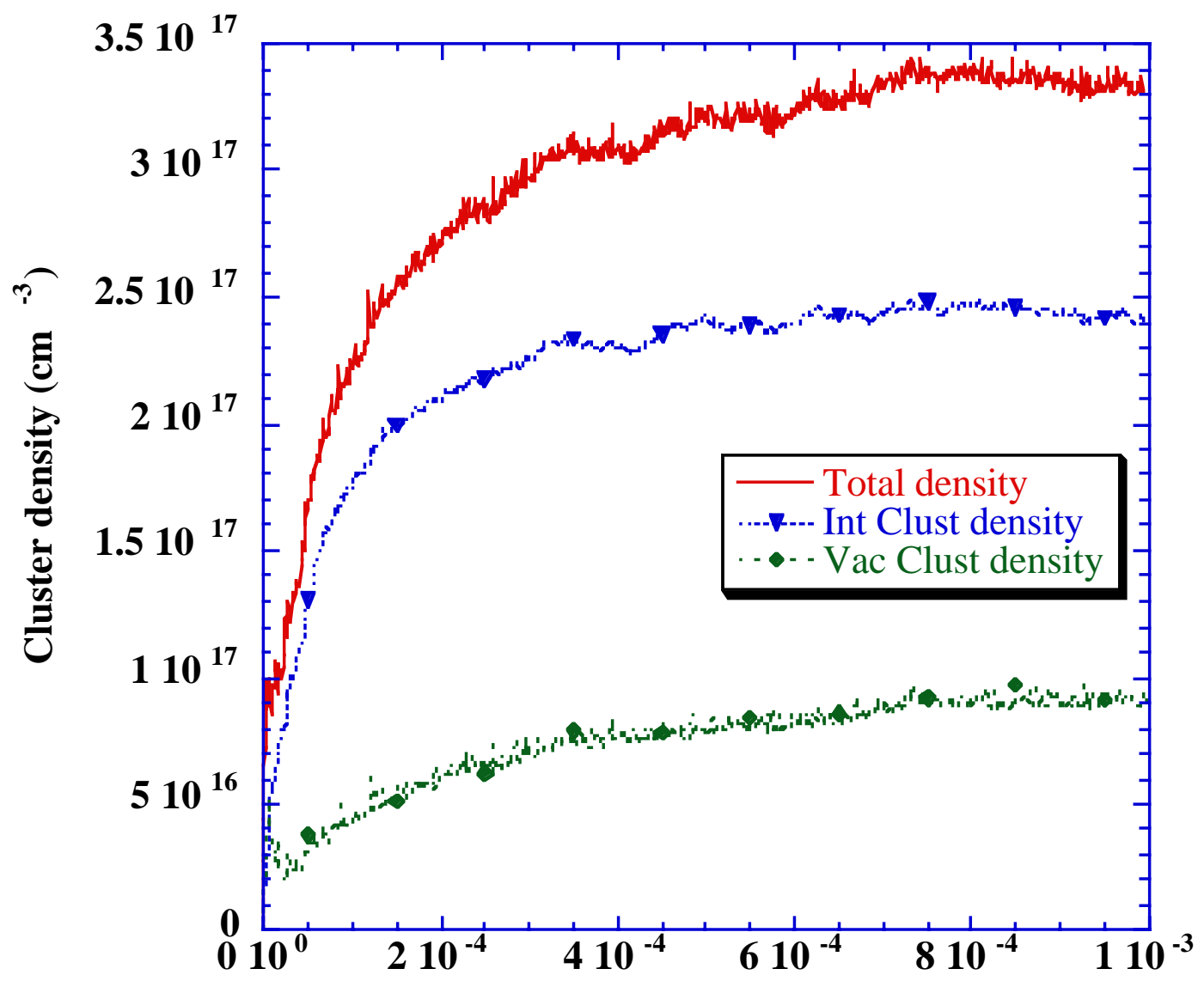

Dose (dpa)

Figure 7. 


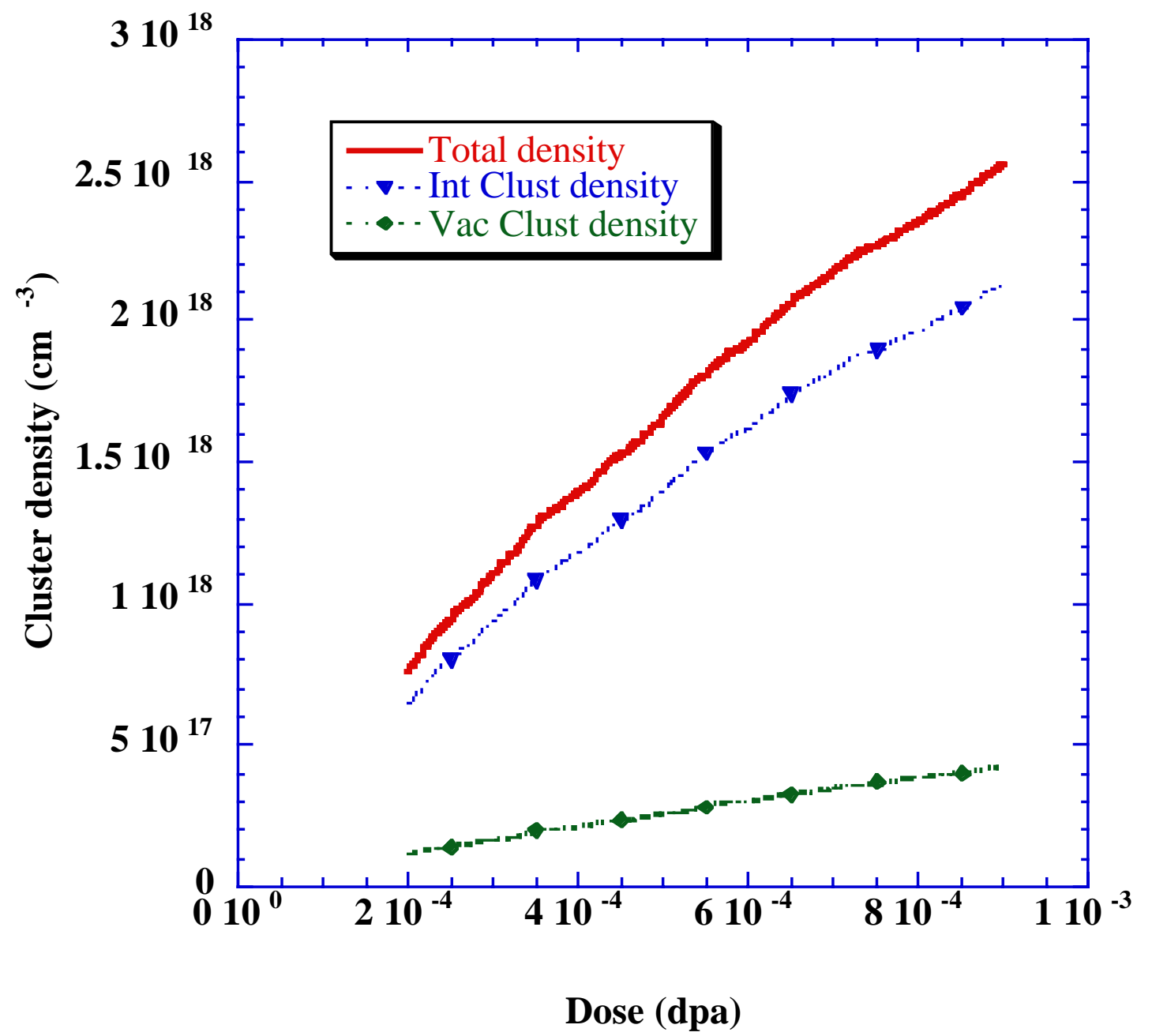

Figure 8. 


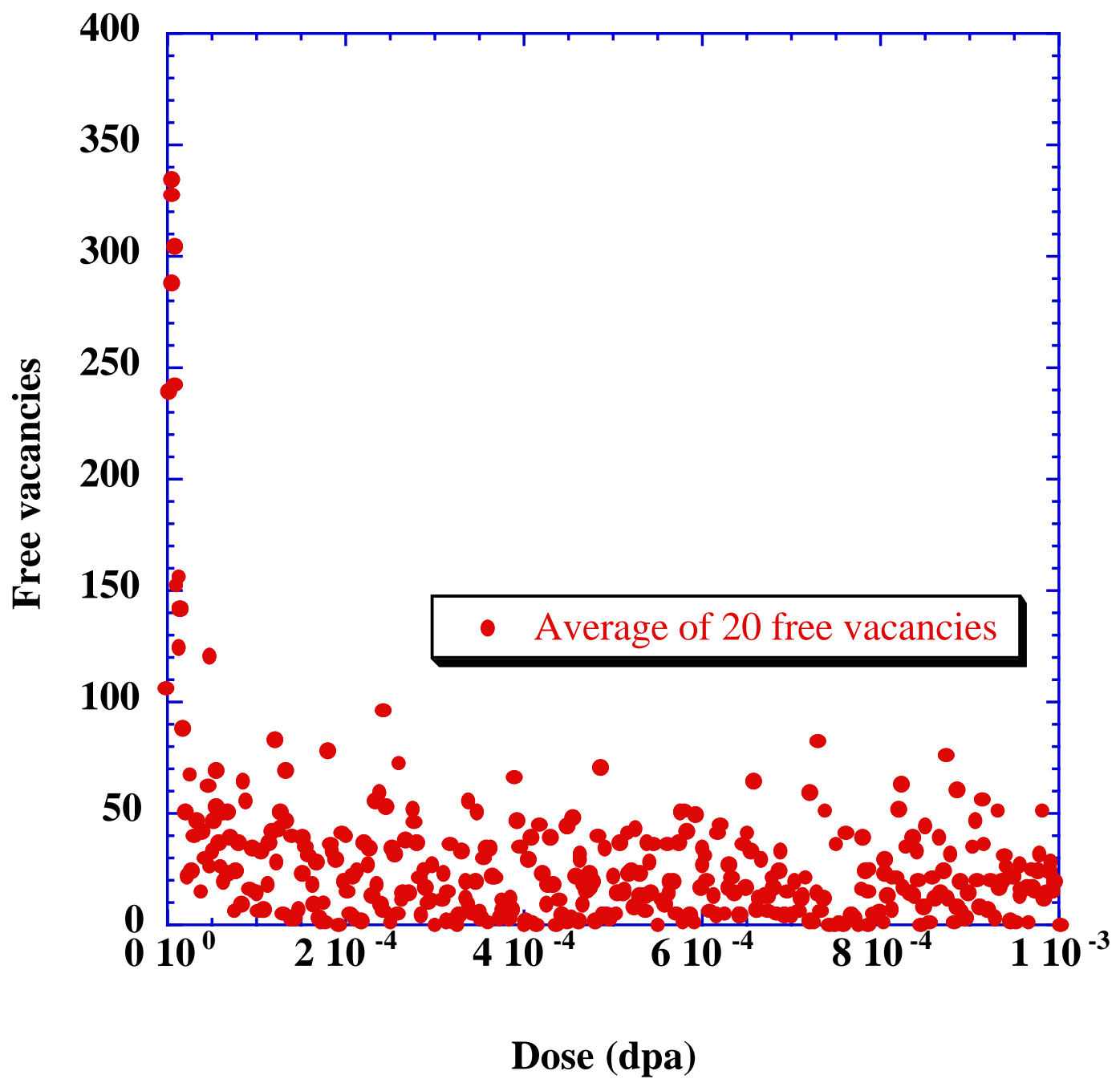

Figure 9. 


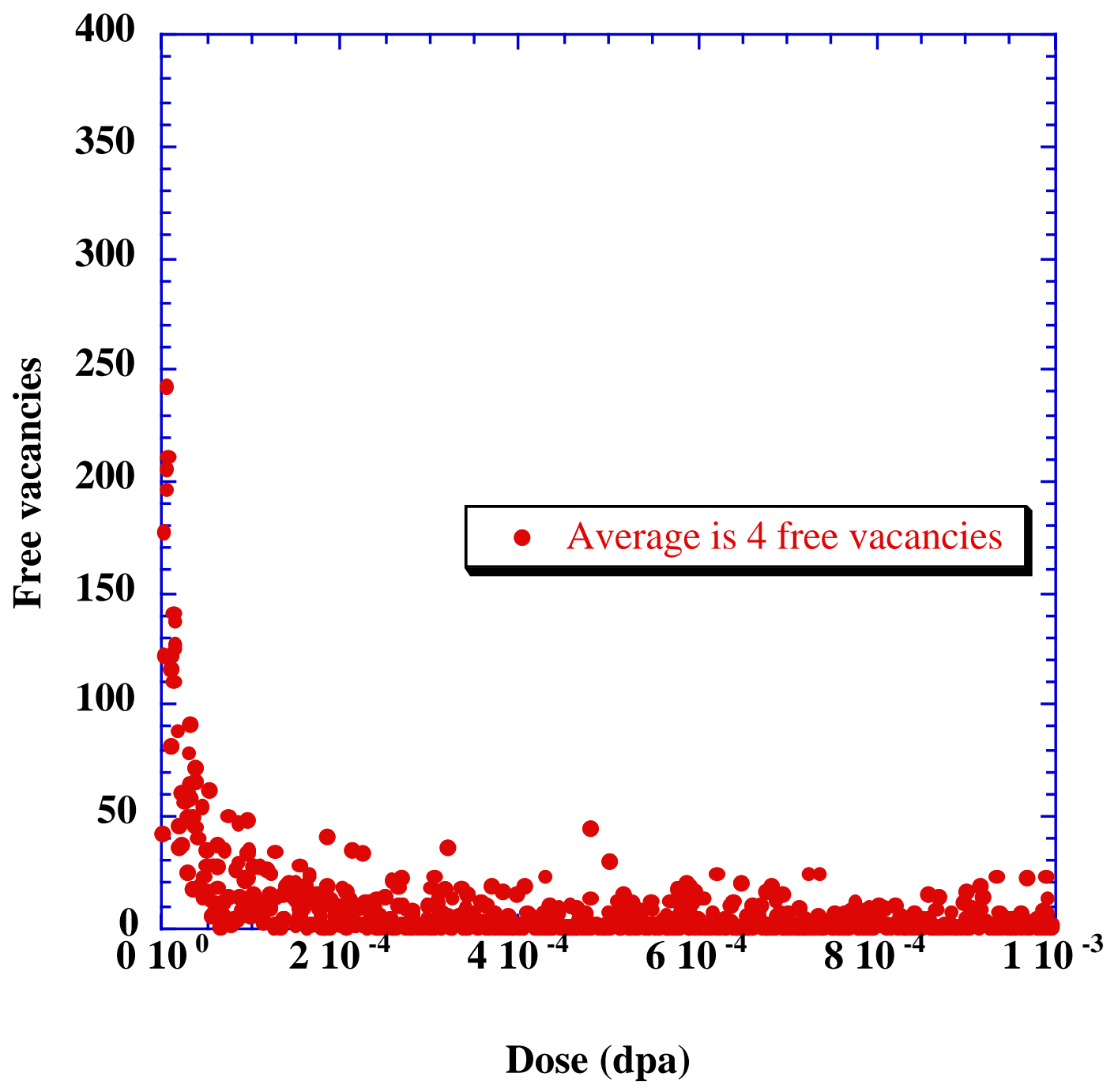

Figure 10. 


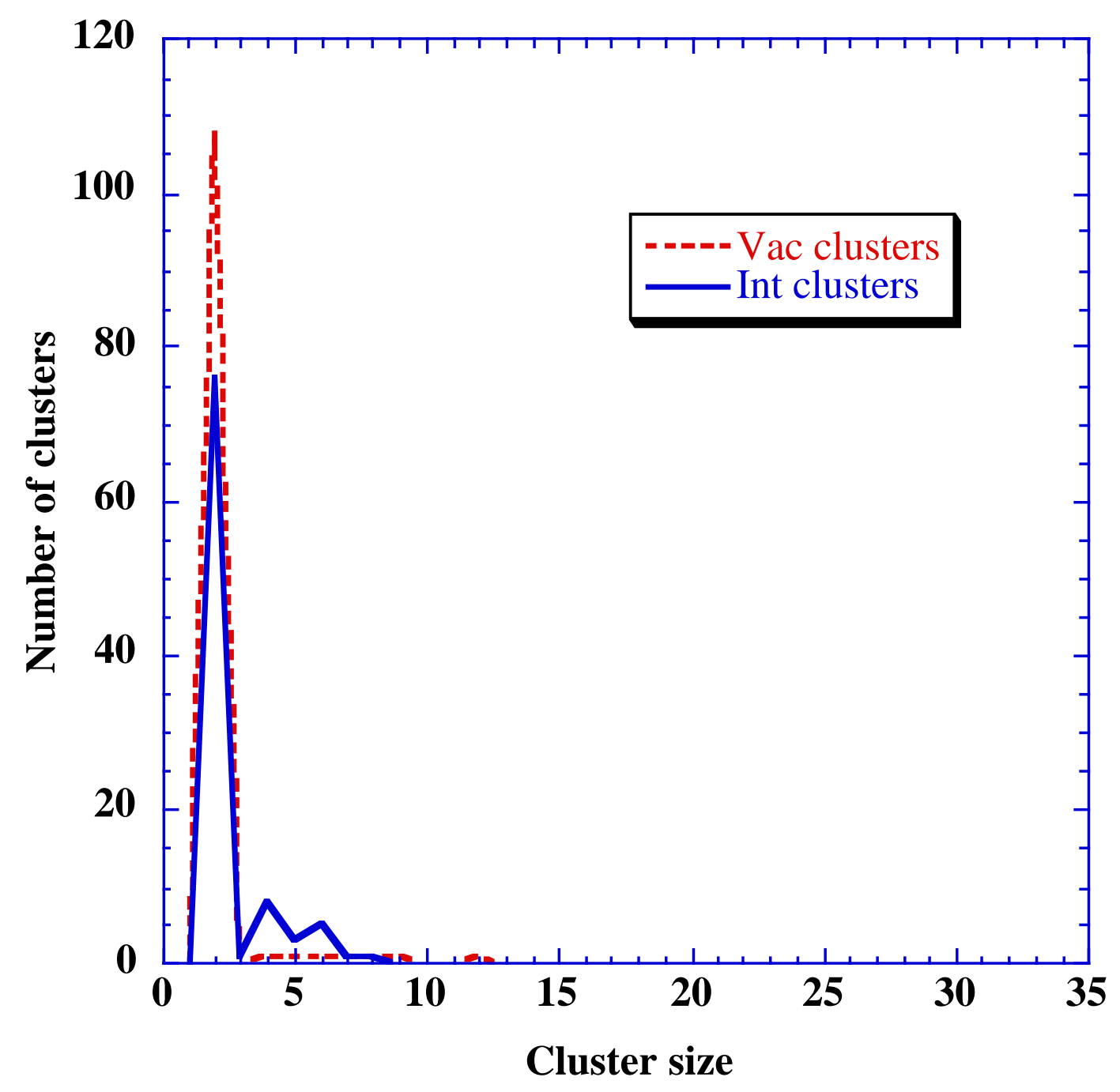

Figure 11. 


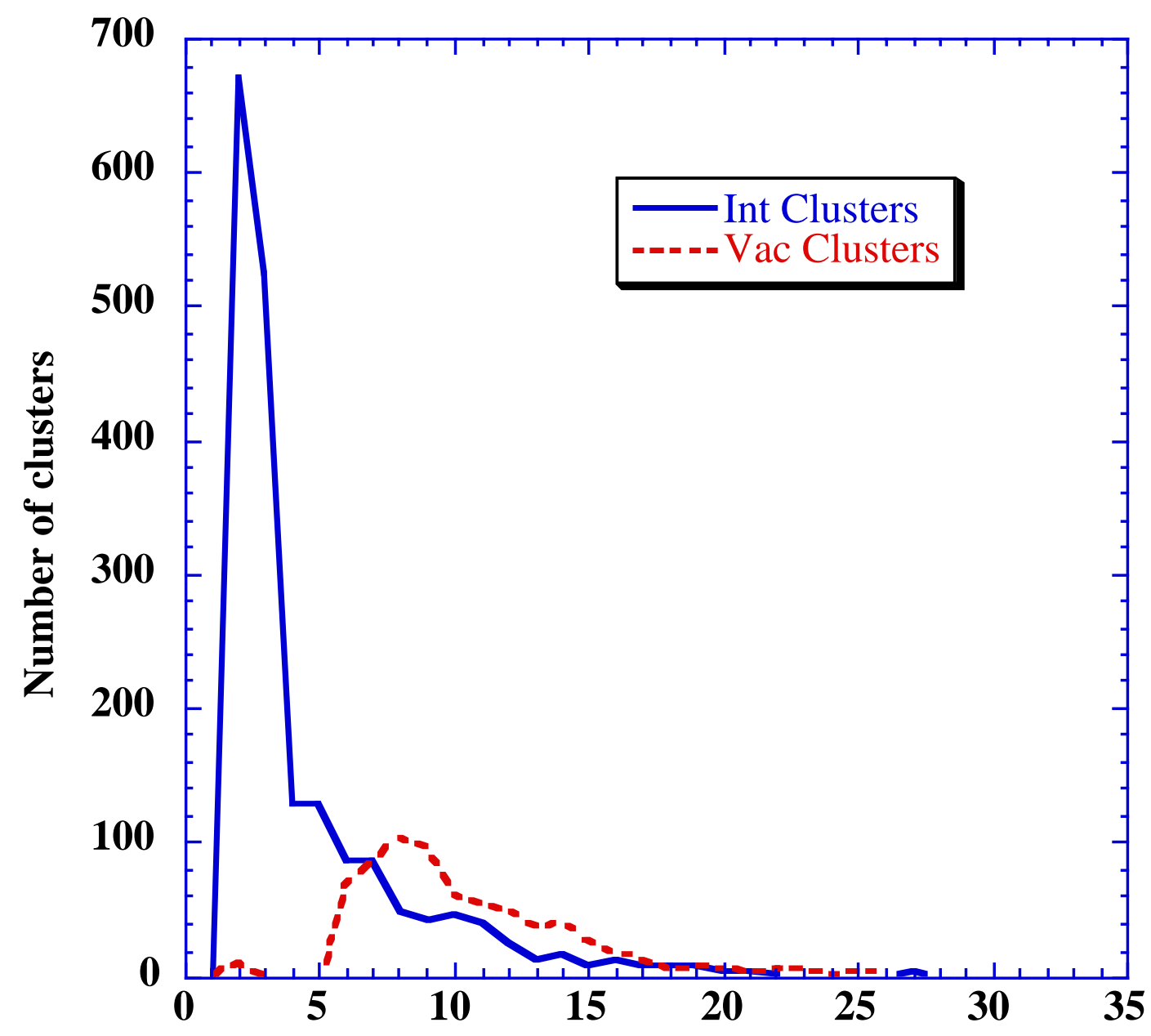

Cluster size 